\title{
Protective effect of higenamine ameliorates collagen-induced arthritis through heme oxygenase-1 and PI3K/Akt/Nrf-2 signaling pathways
}

\author{
WENJIANG DUAN, JIANMIN CHEN, YU WU, YONG ZHANG and YUANSHENG XU \\ Department of Orthopaedics, Jingdu Hospital, Nanjing, Jiangsu 210000, P.R. China
}

Received June 4, 2015; Accepted August 11, 2016

DOI: $10.3892 /$ etm.2016.3730

\begin{abstract}
Existing in Ranunculaceae Aconitum and tomato, with the chemical name 1-phydroxybenzyl-1,2,3,4-tetrahydroisoquinoline, higenamine is widely distributed in China. Higenamine's anti-inflammatory, antioxidant and anti-apoptotic effects have been identified in previous studies. The present study attempted to determine the protective effect of higenamine against collagen-induced arthritis through heme oxygenase-1 (HO-1) and PI3K/Akt/Nrf-2 signaling pathways. A type II collagen (CII)-induced arthritis (CIA) model was established and clinical arthritis scores were used to appraise the curative effect of higenamine. Inflammatory reactions, oxidative damage and caspase-3/9 activation were detected using specific ELISA kits. In addition, western blotting was used to evaluate the expression of $\mathrm{HO}-1$, Akt and $\mathrm{Nrf}-2$ protein in CII-induced CIA mice. In CII-induced CIA mice, the clinical arthritis scores, inflammatory reactions, oxidation damage and caspase-3/9 activation were increased and activated. The results demonstrated that treatment with higenamine significantly reduced the elevation of clinical arthritis scores $(\mathrm{P}<0.01)$, and suppressed the promotion of inflammatory reactions, oxidation damage and caspase-3/9 activation. Furthermore, higenamine significantly increased HO-1 protein expression $(\mathrm{P}<0.01)$ and upregulated the PI3K/Akt/Nrf-2 signal pathway in CII-induced CIA mice. Collectively, it is concluded that higenamine protects against CII-induced CIA through the induction of HO-1 and the upregulation of the $\mathrm{PI} 3 \mathrm{~K} / \mathrm{Akt} / \mathrm{Nrf}-2$ signaling pathway. In conclusion, higenamine may be a beneficial drug for protecting against CIA.
\end{abstract}

Correspondence to: $\mathrm{Mr}$ Jianmin Chen, Department of Orthopaedics, Jingdu Hospital, 34-34 Chang Fu Street, Nanjing, Jiangsu 210000, P.R. China

E-mail: cdgjianminchen@yeah.net

Key words: higenamine, type I collagen-induced arthritis, heme oxygenase-1, phosphatidylinositol-3-kinase/protein kinase B/nuclear factor (erythroid-derived 2)-like 2

\section{Introduction}

Rheumatoid arthritis (RA) is a type of chronic and systemic autoimmunity disease that is characterized by erosive joint synovitis (1). The morbidity of women is 2 fold higher than men (2). The morbidity of RA is $0.01-0.05 \%$ and the nosometry is $0.18-1.07 \%$ in different populations worldwide (2). As a result, RA is a disease which has incapacitated numerous laborers in China (3). According to the epidemiological investigation in the United States, the difference between the mortality rate of patients with RA and healthy individuals is increasing (4). Nosogenesises of RA is currently unclear, but heredity, infection, immunity, endocrine and other factors are possible relevant pathogenic factors $(4,5)$.

Dysfunction of the immune system is the primary cause of RA, but the underlying mechanisms are unclear (6). Animal models must be established to determine the mechanism. There are two types of existing animal models of arthritis; one is adjuvant-induced arthritis and the other is arthritis induced by arthrogenic autoantigen, which is the arthritis induced by collagen (CIA) (7). CIA has been studied frequently, and its clinical symptoms and pathological characteristics are the same as RA (8). As a result, CIA is the most commonly used animal model for RA (8).

Ranunculaceae Aconitum is a traditional Chinese medicine that is used as a long-term cardiotonic drug, whose active constituent is a type of alkaloid named higenamine, which is extracted from Ranunculaceae Aconitum's rhizome $(9,10)$. However, the mechanism underlying the protective effect of higenamine on CIA has, to the best of our knowledge, never been investigated. In the present study, the protective effect and associated underlying mechanism of higenamine against CIA is investigated.

\section{Materials and methods}

Materials. Higenamine was purchased from Sigma-Aldrich (Merck Millipore, Darmstadt, Germany); its chemical structure is presented Fig. 1. Freund's Incomplete Adjuvant, bovine type II collagen, tumor necrosis factor- $\alpha$ (TNF- $\alpha$ ), interleukin-1 $\beta$ (IL-1 $\beta$ ), malondialdehyde (MDA) and glutathione (GSH) ELISA kits were purchased from Tauto Biotech Co., Ltd (Shanghai, China). Caspase-3/9 florometric assay kits were purchased from Sigma-Aldrich (Merck Millipore). 


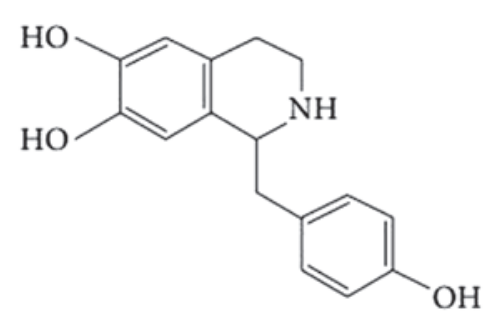

Figure 1. Chemical structure of higenamine.

Bicinchoninic Acid (BCA) assay was purchased from Beyotime Institute of Biotechnology (Nanjing, China).

Animals. Nine week-old male DBA/1J mice (5 weeks-old; weight, 18-20 g) were purchased from Shanghai Cell Bank of Chinese Academy of Sciences (Shanghai, China). The animals were housed in a controlled environment, $23 \pm 1^{\circ} \mathrm{C}$, $12 \mathrm{~h}$ light/dark cycle, relative humidity between 40 and $70 \%$, and provided with standard rodent chow and tap water. All of the animal experiments conducted were performed in compliance with the guidelines and regulations for the use and care of animals established by Jingdu Hospital (Nanjing, China).

Induction of CIA and study design. DBA/1J mice were divided into four groups: Sham; higenamine; CIA model; and CIA + higenamine (each group, $n=6$ ). Sham and higenamine mice were sham-injected with saline or higenamine $(10 \mathrm{mg} / \mathrm{kg})$ for 2 weeks. CIA model and CIA + higenamine mice received injections of Freund's Incomplete Adjuvant with $2 \mathrm{mg} / \mathrm{ml}$ bovine type II collagen at the base of the tail and two sites on the back at 0 and 6 days. Clinically apparent disease onset typically occurs at 10 days. Then, the CIA model and CIA + higenamine mice were sham-injected with saline or higenamine $(10 \mathrm{mg} / \mathrm{kg})$ for 2 weeks.

CIA progression clinical arthritis scores. Arthritis severity was evaluated with a clinical scoring system of 0-4 for each paw: No signs of arthritis, 0; swelling and/or redness in one joint, 1; swelling and/or redness in more than one joint, 2; swelling and/or redness in the entire paw, 3; and severe swelling of the entire paw with deformity and/or ankylosis, 4 . The total arthritis score produced a maximum score of 16 , as the sum of each score of the four limbs.

TNF- $\alpha, I L-1 \beta, M D A, S O D, C A T$ and GSH-PX activities. The blood samples from all mice were permitted to clot for $2 \mathrm{~h}$ were collected via intracardiac puncture after treatment for 2 weeks, and centrifuged at $2,000 \mathrm{xg}$ at $4^{\circ} \mathrm{C}$ for $10 \mathrm{~min}$. The activities of TNF- $\alpha$, IL-1 $\beta$, MDA and GSH were measured using specific ELISA kits in accordance with the manufacturer's instructions.

Caspase-3/9 activities. Under anesthetization by injection of rumpun $(15 \mathrm{mg} / \mathrm{kg})$ and ketamine $(75 \mathrm{mg} / \mathrm{kg})$, the mice were sacrificed using cervical dislocation, and then the arthritis tissue samples from all mice were permitted to clot for $2 \mathrm{~h}$ after treatment for 2 weeks. Then they were homogenized using $100 \mu \mathrm{l}$ tissue lysis buffer (Beyotime Institute of Biotechnology) and a protease inhibitor cocktail

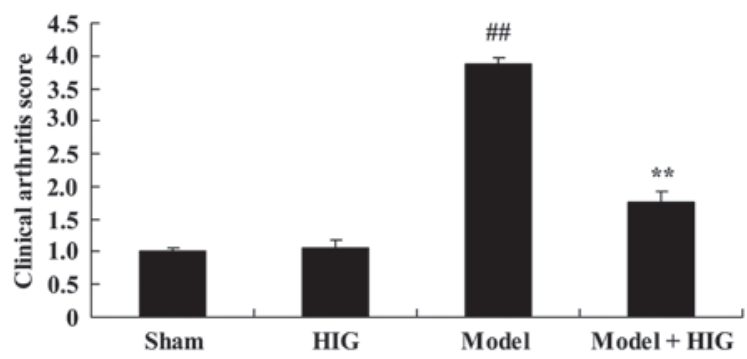

Figure 2. Protective effect of HIG on CIA progression clinical arthritis scores. ${ }^{\# \#} \mathrm{P}<0.01$ vs. the sham group; ${ }^{* *} \mathrm{P}<0.01$ vs. the model group. HIG, higenamine.

(Sigma-Aldrich; Merck Millipore) for $30 \mathrm{~min}$ on ice and centrifuged at $2,000 \mathrm{x}$ g at $4^{\circ} \mathrm{C}$ for $10 \mathrm{~min}$. The concentration of protein was determined using a BCA assay. An equal quantity of protein $(10 \mu \mathrm{g})$ from every sample was separated using the caspase-3/9 florometric assay kits, according to the manufacturer's instructions.

Western blot analysis. The arthritis tissue samples from all mice were permitted to clot for $2 \mathrm{~h}$. Then they were homogenized using $100 \mu \mathrm{l}$ tissue lysis buffer (Beyotime Institute of Biotechnology) and a protease inhibitor cocktail (Sigma-Aldrich; Merck Millipore) for $30 \mathrm{~min}$ on ice and centrifuged at $2,000 \mathrm{x}$ g at $4^{\circ} \mathrm{C}$ for $10 \mathrm{~min}$. The concentration of protein was determined using a BCA assay. An equal quantity of protein $(50 \mu \mathrm{g})$ from every sample was separated by $10-12 \%$ SDS-PAGE and transferred to polyvinylidene floride membranes. The membranes were blocked with $5 \%$ fat-free dry milk in tris-buffered saline with Tween- 20 for $2 \mathrm{~h}$ at room temperature and incubated with anti-HO-1 (1:500; cat. no. sc-136960; Santa Cruz Biotechnology, Inc., Dallas, TX, USA), anti-Akt (1:500; cat. no. sc-271149; Santa Cruz Biotechnology, Inc.), anti-p-Akt (1:500; cat. no. sc-293125; Santa Cruz Biotechnology, Inc.), anti-nuclear factor (erythroid-derived 2)-like 2 (Nrf-2) (cat. no. sc-365949; 1:1,500; Santa Cruz Biotechnology, Inc.) and $\beta$-actin (1:500; cat. no. BB-2116-1; BeastBio, Shanghai, China) overnight at $4^{\circ} \mathrm{C}$. The membranes were then blocked with appropriate secondary antibodies (1:2,000; cat. no. C2221; Applygen Technologies, Inc., Beijing, China) for $1 \mathrm{~h}$ at room temperature and ECL (Applygen Technologies, Inc.) was applied. The blots were quantified using Image $\mathbf{J}$ software (National Institutes of Health, Bethesda, MD, USA).

Statistical analysis. Data were expressed as the mean \pm standard error and analyzed by Student's t-test or analysis of variance with post-hoc (Bonferroni) correction for multiple comparisons, or one-way analysis of variance for multiple comparisons. SPSS 17.0 (SPSS, Inc., Chicago, IL. USA) was used to analyze the data. $\mathrm{P}<0.05$ was considered to indicate a statistically significant difference.

\section{Results}

Protective effect of higenamine on CIA progression clinical arthritis scores. In order to evaluate the protective effect of higenamine on CIA mice, the progression clinical arthritis scores were evaluated. The results demonstrate that clinical 
A

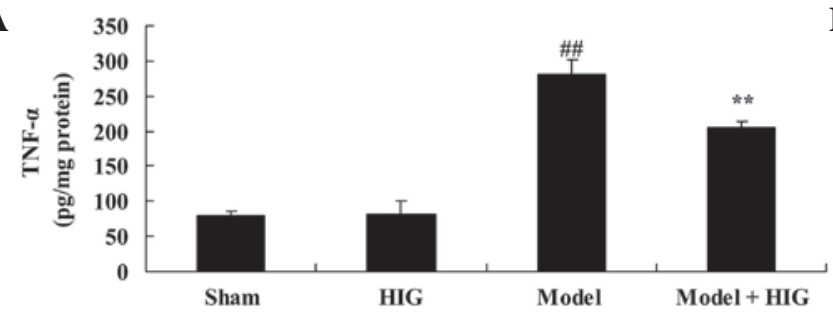

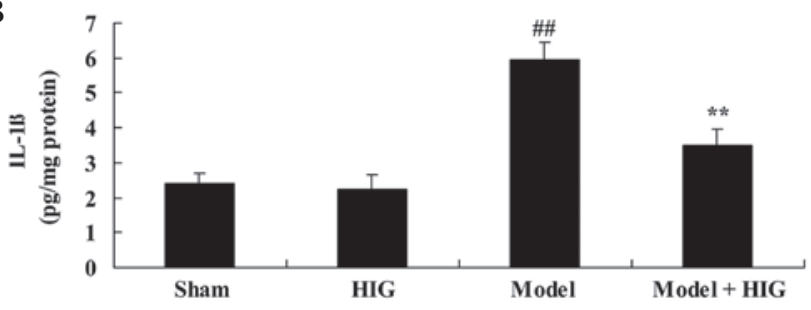

Figure 3. Protective effect of HIG on the (A) TNF- $\alpha$ and (B) IL-1 $\beta$ activities. ${ }^{\# \#} \mathrm{P}<0.01$ vs. the sham group, ${ }^{* *} \mathrm{P}<0.01$ vs. the model group. HIG, higenamine; TNF- $\alpha$, tumor necrosis factor- $\alpha$; IL-1 $\beta$, interleukin- $1 \beta$.

A

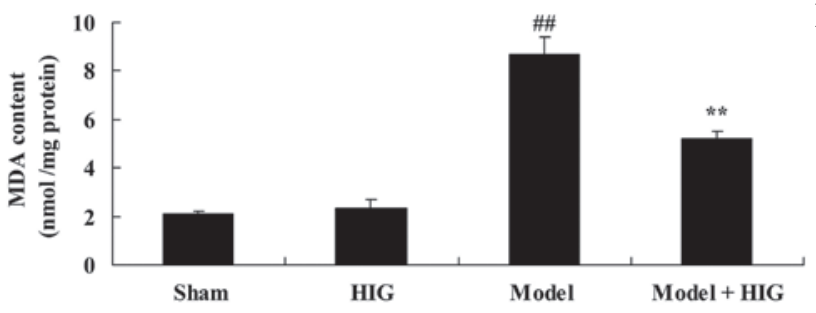

B

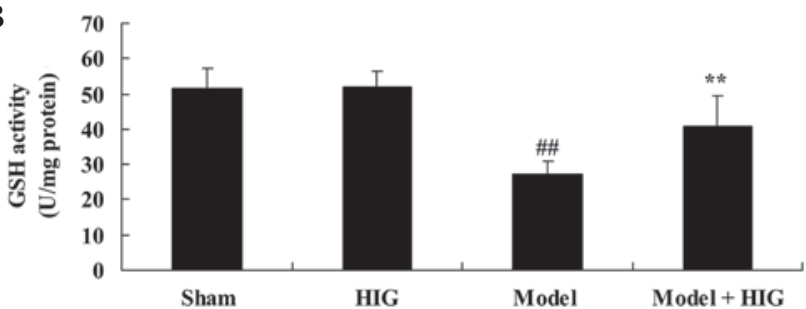

Figure 4. Protective effect of HIG on the (A) MDA and (B) GSH activities. ${ }^{\# \#} \mathrm{P}<0.01$ vs. the sham group, ${ }^{* *} \mathrm{P}<0.01$ vs. the model group. HIG, higenamine; MDA, malondialdehyde; GSH, glutathione.

A

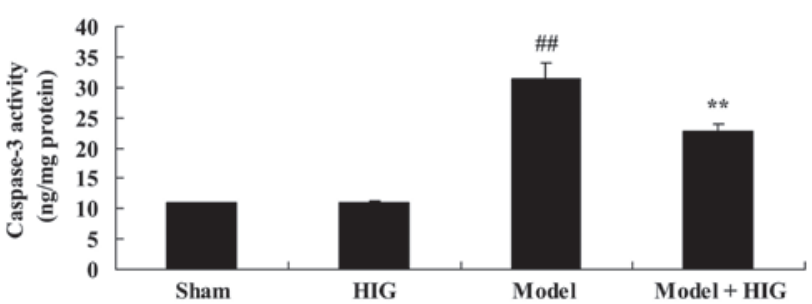

B

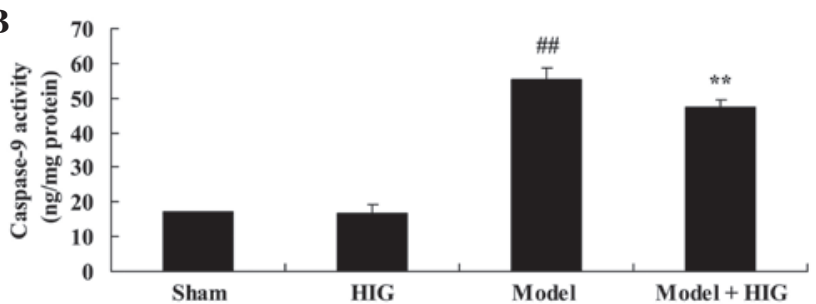

Figure 5. Protective effect of HIG on the (A) caspase-3 and (B) caspase-9 activities. ${ }^{\# \prime} \mathrm{P}<0.01$ vs. the sham group, ${ }^{* * *} \mathrm{P}<0.01$ vs. the model group. HIG, higenamine.

arthritis scores of the sham group were similar to those of the higenamine group (Fig. 2). The clinical arthritis scores of CIA mice were significantly higher compared with those of the sham group $(\mathrm{P}<0.01$; Fig. 2). The progression of clinical arthritis scores of CIA mice were significantly reduced by treatment with higenamine $(\mathrm{P}<0.01$; Fig. 2$)$.

Protective effect of higenamine on the TNF- $\alpha$ and IL-1 $\beta$ activities. To determine the mechanism by which higenamine treatment exerts an anti-inflammatory effect on CIA mice, the activities of TNF- $\alpha$ and IL-1 $\beta$ were measured. TNF- $\alpha$ and IL-1 $\beta$ activities in sham group mice were similar to those of mice in the higenamine group (Fig. 3). CIA mice had significantly increased inflammation factors compared with mice in the sham group $(\mathrm{P}<0.01$; Fig. 3$)$. However, treatment with higenamine significantly reduced these CIA-induced inflammation factors in CIA mice $(\mathrm{P}<0.01$; Fig. 3$)$.

Protective effect of higenamine on $T M D A$ and GSH activities. To investigate the mechanism of higenamine on oxidative stress in CIA mice, MDA and GSH activities were measured in each group. The MDA and GSH activities of sham mice were similar to those in mice in the higenamine group (Fig. 4). Compared with the sham group, CIA significantly increased the MDA activity and suppressed GSH activity in CIA mice
$(\mathrm{P}<0.01$; Fig. 4). Meanwhile, higenamine treatment significantly recovered abnormal MDA and GSH activities in CIA mice $(\mathrm{P}<0.01$; Fig. 4).

Protective effect of higenamine on caspase-3/9 activities. In order to ascertain the anti-apoptotic effect of higenamine on CIA mice, the caspase-3/9 activities were detected in different groups. The caspase-3/9 activities of the sham group were equipotent to those of mice in the higenamine group (Fig. 5). There was a significant increase in caspase-3/9 activities of CIA mice without higenamine treatment (Fig. 5). However, pretreatment with higenamine significantly weakened CIA-induced caspase-3/9 activities of CIA mice, as compared with the CIA model group ( $\mathrm{P}<0.01$; Fig. 5).

Protective effect of higenamine on $\mathrm{HO}-1$ protein expression. To investigate the mechanisms underlying the effect of higenamine on CIA mice, HO-1 protein expression was measured using western blot analysis. The results demonstrate that HO-1 protein expression in the sham group was similar to that of the higenamine group (Fig. 6). Fig. 6 clearly demonstrated that HO-1 protein expression in the CIA was significantly lower compared with that in the sham group $(\mathrm{P}<0.01)$. In addition, higenamine significantly enhanced the suppression of $\mathrm{HO}-1$ protein expression in CIA mice $(\mathrm{P}<0.01$; Fig. 6). 
A

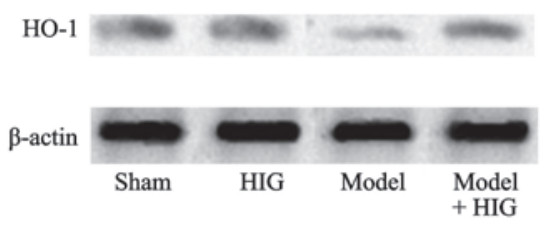

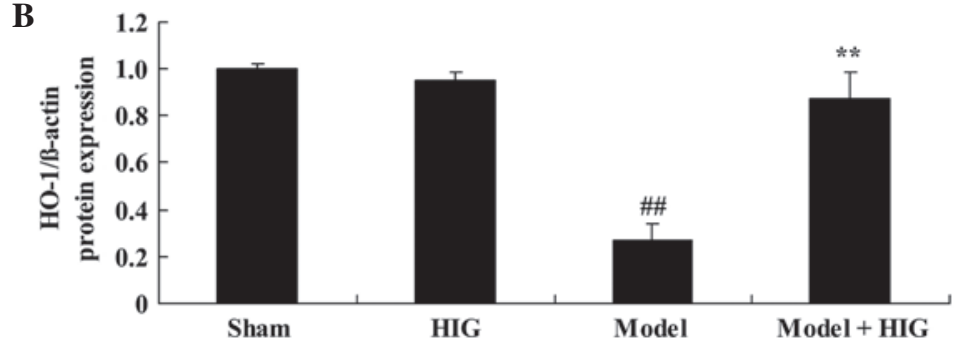

Figure 6. Protective effect of HIG on the HO-1 protein expression by (A) western blotting assays and (B) statistical analysis of HO-1 protein expression. ${ }^{\# /} \mathrm{P}<0.01$ vs. the sham group, ${ }^{* *} \mathrm{P}<0.01$ vs. the model group. HIG, higenamine; HO-1, heme-oxygenase-1.

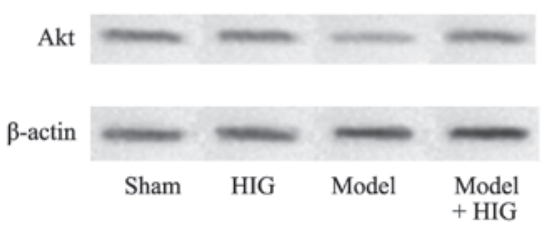

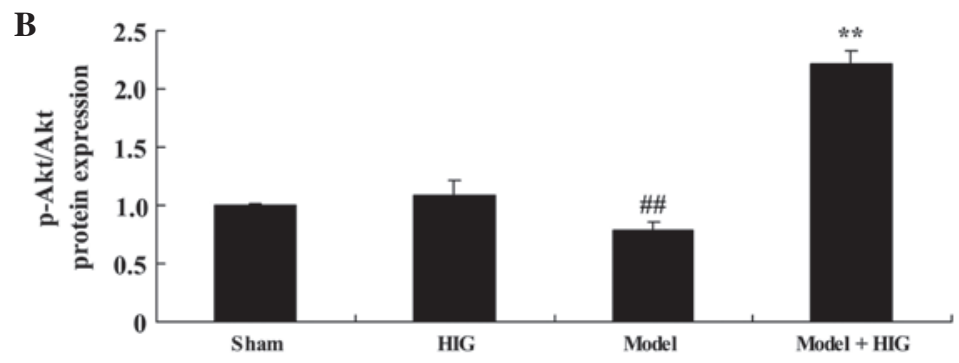

Figure 7. Protective effect of HIG on the p-Akt/Akt protein expression by (A) western blotting assays and (B) statistical analysis of p-Akt/Akt protein expression. ${ }^{\# \#} \mathrm{P}<0.01$ vs. the sham group, ${ }^{* *} \mathrm{P}<0.01$ vs. the model group. HIG, higenamine; $\mathrm{p}$-Akt, phosphorylated Akt.

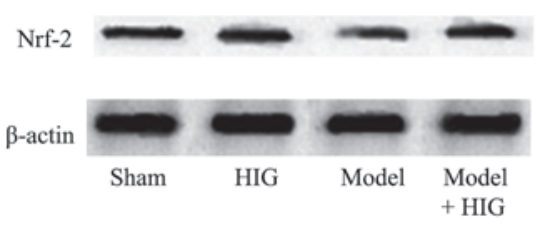

B

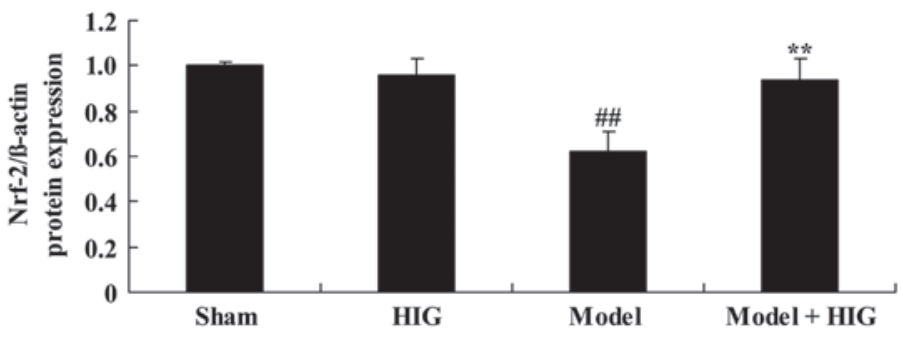

Figure 8. Protective effect of HIG on the Nrf-2 protein expression by (A) western blotting assays and (B) statistical analysis of Nrf-2 protein expression. ${ }^{\# \#} \mathrm{P}<0.01$ vs. the sham group, ${ }^{* *} \mathrm{P}<0.01$ vs. the model group. HIG, higenamine; Nrf-2, nuclear factor (erythroid-derived 2 )-like 2.

Protective effect of higenamine on Akt protein expression. To confirm the effect of higenamine on Akt protein expression, western blot analysis was used to analyze the Akt and p-Akt protein expression in CIA mice. No significant changes in $\mathrm{p}$-Akt/Akt protein expression between the sham and higenamine group were identified (Fig. 7). CIA significantly suppressed p-Akt/Akt protein expression of CIA mice, compared with the sham group $(\mathrm{P}<0.01$; Fig. 7). In addition, higenamine significantly activated the suppression of p-Akt/Akt protein expression in CIA mice ( $\mathrm{P}<0.01$; Fig. 7).

Protective effect of higenamine on Nrf-2 protein expression. To investigate the mechanisms underlying the effect of higenamine on CIA mice, Nrf-2 protein expression was observed using western blot analysis. It was identified that Nrf-2 protein expression in the sham group was similar to that in the higenamine group (Fig. 8). CIA mice had significantly reduced Nrf-2 protein expression compared with that in the sham group $(\mathrm{P}<0.01$; Fig. 8). As expected, Nrf-2 protein expression was significantly increased by treatment with higenamine in CIA mice $(\mathrm{P}<0.05$; Fig. 8$)$.

\section{Discussion}

RA is a type of chronic and systemic autoimmune disease that is characterized by erosive joint synovitis, which harms body systems and organs, such as joints, the heart and the kidney (11). RA attacks facet joints symmetrically, for instance, articulationes digitorum manus, articulationes digitorum pedis and wrist joints (12). In the advanced stage of RA, ankylosis, malformation and dysfunction of these facet joints will arise. In addition, fever, anemia, scleritis and pericarditis are likely to develop (13). In the present study, treatment with higenamine effectively inhibited the promotion of clinical arthritis scores of CIA mice.

According to a previous pathological study, histopathologic features of RA are pannus formation and destruction of cartilage and bone, as well as leukopedesis and lymphocyte infiltration (particularly T-lymphocytes) in affected articular tissue (14). The high expression level of IL-1 $\beta$ and TNF- $\alpha$ in the blood of patients with RA suggests that these two factors are associated with RA. Secreted by synovial cells, in autocrine and paracrine forms, TNF- $\alpha$ can promote inflammatory mediator secretion, such as prostaglandin E2 (PGE2), by which 
inflammation will be stimulated and the cartilago articularis will be destroyed (15). As a result, TNF- $\alpha$ becomes a factor reflecting the activity and severity of RA. IL- $1 \beta$, another inflammatory mediator, is secreted by monocyte-macrophages, and can induce synovial and cartilage cells to produce collagenase and PGE2, which leads to inflammation of the synovium and disintegration of the mesochondrium (16). At the same time, PGE2 can stimulate neutrophil, macrophage and lymphocyte aggregation in the articular cavity so that the arthritis will be exacerbated, which is a vicious cycle. The results of the present study demonstrated that higenamine effectively reduces CIA-induced TNF- $\alpha$ and IL- $1 \beta$ activities in CIA mice. Ha et al (17) reported that higenamine reduces HMGB1 during hypoxia-induced brain injury by the induction of inflammation.

MDA is an end product of lipid oxidation, the expression level of which can reflect the severity of oxidative damage. According to clinical research, the peroxide levels of patients with RA is raised and their antioxidant capacity is reduced, suggesting that there is a higher MDA expression level in patients with RA compared with healthy individuals. GSH can protect cells from oxidative damage, so the expression level of GSH can reflect the state of RA. Individuals with lower activity levels of GSH have a lower antioxidative ability. The present study demonstrated that pretreatment with higenamine significantly recovered abnormal CIA-induced MDA and GSH activities in CIA mice. Lee et al (18) concluded that higenamine reduces apoptotic cell death through suppression of oxidation in rat myocardial ischemia-reperfusion injury.

Different from necrosis, apoptosis is an active process which is concerned with gene activation, expression and control. Mitochondria are stimulated by numerous signals and when their functions are depressed they eventually release apoptotic factors. Caspase is activated by the mitochondria's morphologic and functional change, which results in the release of cytochrome $c$. The present data clearly demonstrate that higenamine significantly weakens CIA-induced caspase-3/9 activities of CIA mice. In addition, Lee et al (18) concluded that higenamine reduces apoptotic cell death through suppression of oxidation and caspase-3 activity in rat myocardial ischemia-reperfusion injury.

As one of the rate-limiting enzymes of the heme oxygenase, HO-1 expression will be increased in stress, which is associated with the combination of NF- $\mathrm{BB}$ and genomic accessible sites of HO-1 (19). HO-1 is involved in anti-inflammatory, antioxidant and anti-apoptosis pathways (20). Research aimed at evaluating the possibility of using HO-1 to diagnose RA has demonstrated that $\mathrm{HO}-1$, which is potentially associated with RA, is found in the synovial tissues of patients with RA (20). This suggests that higenamine significantly enhanced the suppression of HO-1 protein expression of CIA mice. Zhang et al (21) reported that higenamine promotes M2 macrophage activation via induction of HO-1 in spinal cord injury.

As one of the important intracellular transduction signal pathways, the PI3K/Akt signaling pathway regulates cell division, differentiation, apoptosis and other cell activities (22). The loss and activation failure of $\mathrm{Nrf}-2$ can result in the increase of sensibility to stressors, the extension of inflammation repair time and the promotion of cancer and cell apoptosis (23). HePG2 cells are stimulated by capsaicin to increase the expression of
HO-1, and this is achieved by increasing the nuclear translocation of Nrf-2 to activate the PI3K/Akt signaling pathway (24). This regulation of activation serves an important role in the induction and maintenance of the expression of metabolizing and antioxidant enzymes. In addition, this regulation can protect cells from oxidative injury (25). In the present study, it was demonstrated that higenamine activates the suppression of p-Akt/Akt and Nrf-2 protein expression in CIA mice. In addition, Ha et al (17) demonstrated that higenamine reduces HMGB1 via the PI3K/Akt/Nrf-2 signaling pathway in rats with hypoxia-induced brain injury.

In conclusion, the current study demonstrates that the protective effect of higenamine ameliorates collagen-induced arthritis through the induction of HO-1 and PI3K/Akt/Nrf-2 signaling pathways. Thus, higenamine may be an important novel therapeutic agent for the treatment of CIA.

\section{Acknowledgements}

This work was supported by Nanjing city science and technology project (210303089).

\section{References}

1. Bae J and Park JW: Topical delivery of leflunomide for rheumatoid arthritis treatment: Evaluation of local tissue deposition of teriflunomide and its anti-inflammatory effects in an arthritis rat model. Drug Dev Ind Pharm 27: 1-9, 2015 (Epub ahead of print).

2. Smolen JS, Weinblatt ME, van der Heijde D, Rigby WF, van Vollenhoven R, Bingham CO III, Veenhuizen M, Gill A, Zhao F, Komocsar WJ, et al: Efficacy and safety of tabalumab, an anti-B-cell-activating factor monoclonal antibody, in patients with rheumatoid arthritis who had an inadequate response to methotrexate therapy: results from a phase III multicentre, randomised, double-blind study. Ann Rheum Dis 74: 1567-1570, 2015.

3. Westhovens R, Robles M, Ximenes AC, Wollenhaupt J, Durez P, Gomez-Reino J, Grassi W, Haraoui B, Shergy W, Park SH, et al: Maintenance of remission following 2 years of standard treatment then dose reduction with abatacept in patients with early rheumatoid arthritis and poor prognosis. Ann Rheum Dis 74: 564-568, 2015.

4. Chung KC, Kotsis SV, Fox DA, Regan M, Burke FD, Wilgis EF and Kim HM: Differences between the United States and the United Kingdom in the treatment of rheumatoid arthritis: Analyses from a hand arthroplasty trial. Clin Rheumatol 29: 363-367, 2010.

5. Kavanaugh A, St Clair EW, McCune WJ, Braakman T and Lipsky P: Chimeric anti-tumor necrosis factor-alpha monoclonal antibody treatment of patients with rheumatoid arthritis receiving methotrexate therapy. J Rheumatol 27: 841-850, 2000.

6. Richter J, Capková K, Hř́ibalová V, Vannucci L, Danyi I, Malý M and Fišerová A: Collagen-induced arthritis: Severity and immune response attenuation using multivalent $\mathrm{N}$-acetyl glucosamine. Clin Exp Immunol 177: 121-133, 2014.

7. Kamada H, Goto M, Matsuura S, Takaoka Y and Nagai H: Immunopharmacological studies on collagen-induced arthritis in dark Agouti (DA) rats. Jpn J Pharmacol 74: 313-322, 1997.

8. Pimentel TA, Sampaio AL, D'Acquisto F, Perretti M and Oliani SM: An essential role for mast cells as modulators of neutrophils influx in collagen-induced arthritis in the mouse. Lab Invest 91: 33-42, 2011.

9. Bonamore A, Barba M, Botta B, Boffi A and Macone A: Norcoclaurine synthase: Mechanism of an enantioselective pictet-spengler catalyzing enzyme. Molecules 15: 2070-2078, 2010.

10. Feng S, Jiang J, Hu P,Zhang JY, Liu T, Zhao Q and Li BL: A phase I study on pharmacokinetics and pharmacodynamics of higenamine in healthy Chinese subjects. Acta Pharmacol Sin 33: 1353-1358, 2012.

11. Wang Z, Chen Z, Yang S, Wang Y, Yu L, Zhang B, Rao Z, Gao J and Tu S: (1)H NMR-based metabolomic analysis for identifying serum biomarkers to evaluate methotrexate treatment in patients with early rheumatoid arthritis. Exp Ther Med 4: 165-171, 2012. 
12. Luz KR, Furtado RN, Nunes CC, Rosenfeld A, Fernandes AR and Natour J: Ultrasound-guided intra-articular injections in the wrist in patients with rheumatoid arthritis: A double-blind, randomised controlled study. Ann Rheum Dis 67: 1198-1200, 2008.

13. Nishiya K, Hisakawa N, Tahara K, Matsumori A, Ito H, HashimotoK, Nakatani Kand Takatori K: Additive triple DMARD combination therapy of a low dose of sulfhydryl compounds, sulfasalazine and methotrexate in the treatment of rheumatoid arthritis: A clinical trial. Acta Med Okayama 53: 275-279, 1999.

14. Wislowska M and Jakubicz D: Preliminary evaluation in rheumatoid arthritis activity in patients treated with TNF-alpha blocker plus methotrexate versus methotrexate or leflunomide alone. Rheumatol Int 27: 641-647, 2007.

15. Huang C, Wan L and Liu J: Effect of Xinfeng capsule on nuclear factor Kappa B/tumor necrosis factor alpha and transforming growth factor beta $1 /$ Smads pathways in rats with cardiac injuries induced by adjuvant arthritis. J Tradit Chin Med 36: 92-100, 2016

16. Ma Z, Piao T, Wang Y and Liu J: Astragalin inhibits IL-1betainduced inflammatory mediators production in human osteoarthritis chondrocyte by inhibiting NF-kappaB and MAPK activation. Int Immunopharmacol 25: 83-87, 2015.

17. Ha YM, Kim MY, Park MK, Lee YS, Kim YM, Kim HJ, Lee JH and Chang KC: Higenamine reduces HMGB1 during hypoxia-induced brain injury by induction of heme oxygenase-1 through PI3K/Akt/Nrf-2 signal pathways. Apoptosis 17: 463-474, 2012.

18. Lee YS, Kang YJ,Kim HJ, Park MK, Seo HG, Lee JH, Yun-Choi HS and Chang KC: Higenamine reduces apoptotic cell death by induction of heme oxygenase-1 in rat myocardial ischemia-reperfusion injury. Apoptosis 11: 1091-1100, 2006.
19. Chi PL, Liu CJ, Lee IT, Chen YW, Hsiao LD and Yang CM: HO-1 induction by CO-RM2 attenuates TNF- $\alpha$-induced cytosolic phospholipase A2 expression via inhibition of PKC $\alpha$-dependent NADPH oxidase/ROS and NF-кB. Mediators Inflamm 2014: 279171, 2014.

20. Kirino Y, Takeno M, Murakami S, Kobayashi M, Kobayashi H, Miura K, Ideguchi H, Ohno S, Ueda A and Ishigatsubo Y: Tumor necrosis factor alpha acceleration of inflammatory responses by down-regulating heme oxygenase 1 in human peripheral monocytes. Arthritis Rheum 56: 464-475, 2007.

21. Zhang Z, Li M, Wang Y, Wu J and Li J: Higenamine promotes M2 macrophage activation and reduces Hmgbl production through HO-1 induction in a murine model of spinal cord injury. Int Immunopharmacol 23: 681-687, 2014

22. Torres-Arzayus MI, Font de Mora J, Yuan J, Vazquez F, Bronson R, Rue M, Sellers WR and Brown M: High tumor incidence and activation of the PI3K/AKT pathway in transgenic mice define AIB1 as an oncogene. Cancer Cell 6: 263-274, 2004.

23. Pareek TK, Belkadi A, Kesavapany S, Zaremba A, Loh SL, Bai L, Cohen ML, Meyer C, Liby KT, Miller RH, et al: Triterpenoid modulation of IL-17 and Nrf-2 expression ameliorates neuroinflammation and promotes remyelination in autoimmune encephalomyelitis. Sci Rep 1: 201, 2011.

24. Ha do T, Oh J, Khoi NM, Dao TT, Dung V, Do TN, Lee SM, Jang TS, Jeong GS and Na M: In vitro and in vivo hepatoprotective effect of ganodermanontriol against t-BHP-induced oxidative stress. J Ethnopharmacol 150: 875-885, 2013.

25. Ugur M, Yildirim K, Kiziltunc A, Erdal A, Karatay S and Senel K: Correlation between soluble intercellular adhesion molecule 1 level and extracellular superoxide dismutase activity in rheumatoid arthritis: A possible association with disease activity. Scand J Rheumatol 33: 239-243, 2004. 